\title{
Single arterial occlusion to locate resistance in patients with pulmonary hypertension
}

\author{
P. Fesler*, A. Pagnamenta*, J-L. Vachiéry*, S. Brimioulle*, S. Abdel Kafi*, A. Boonstra\#, \\ M. Delcroix ${ }^{\top}$, R.N. Channick ${ }^{+}$, L.J. Rubin ${ }^{+}$, R. Naeije*
}

Single arterial occlusion to locate resistance in patients with pulmonary hypertension. P. Fesler, A. Pagnamenta, J-L. Vachiéry, S. Brimioulle, S. Abdel Kafi, A. Boonstra, M. Delcroix, R.N. Channick, L.J. Rubin, R. Naeije. (C)ERS Journals Ltd 2003.

ABSTRACT: The purpose of this study was to determine the site of increased resistance using the arterial occlusion technique in patients with severe pulmonary hypertension.

Pulmonary vascular resistance was partitioned in arterial and venous components based on double exponential fitting analysis of the pulmonary artery pressure decay curve: after balloon occlusion in 36 patients with pulmonary arterial hypertension (PAH); at baseline and during the inhalation of 20 parts per million of nitric oxide (NO); in four patients with chronic thromboembolic pulmonary hypertension; and in two patients with pulmonary veno-occlusive disease.

In the patients with $\mathrm{PAH}$, at baseline, mean pulmonary artery pressure was $56 \pm 2 \mathrm{mmHg}$ (mean $\pm \mathrm{SE}$ ), with an arterial component of resistance of $63 \pm 1 \%$. Inhaled NO did not change the partition of resistance. The arterial component of resistance amounted on average to $42 \%$ and $77 \%$ in the patients with veno-occlusive disease and the patients with thromboembolic pulmonary hypertension, respectively. However, the partitioning of resistance did not discriminate between these three diagnostic categories.

The occlusion technique may help to locate the predominant site of increased resistance in patients with severe pulmonary hypertension, but does not allow for a satisfactory differential diagnosis on an individual basis.

Eur Respir J 2003; 21: 31-36.
*Depts of Physiology, Cardiology and Intensive Care, Erasme University Hospital Brussels, Brussels, Belgium. " Dept of Pneumology, Academic Hospital of the Free University of Amsterdam, Amsterdam, the Netherlands. Dept of Pneumology, Gasthuisberg University Hospital, Leuven, Belgium. ${ }^{+}$Pulmonary and Critical Care Division, UCSD Medical Center, San Diego, CA, USA.

Correspondence: R. Naeije, Laboratory of Physiology, Erasmus Campus, CP 604, Route de Lennik 808, B-1070 Brussels, Belgium.

Fax: 3225554124

E-mail: rnaeije@ulb.ac.be

Keywords: Chronic thromboembolic pulmonary hypertension, effective pulmonary capillary pressure, nitric oxide, pulmonary arterial hypertension, pulmonary vascular resistance, pulmonary veno-occlusive disease

Received: June 242002

Accepted after revision: August 192002

Supported by grant number 3.4567 .00 from the Fonds de la Recherche Scientifique Médicale, Brussels, Belgium. P. Fesler was a fellow of the Erasmus Foundation, Brussels, Belgium.
The occlusion technique can be used in intact animals and patients for the partitioning of pulmonary vascular resistance (PVR) into an arterial segment (PVRa) and a capillary-venous segment, and for the determination of an effective pulmonary capillary pressure $\left(P_{\mathrm{c}}\right)[1,2]$. The method identified a hydrostatic mechanism, with an important role accounting for early high-altitude pulmonary oedema [3]. The single arterial occlusion technique applied to a small series of patients with primary pulmonary hypertension (PPH) showed a longitudinal distribution of resistance that appeared similar to that found in normal pulmonary circulation, with a PVRa $\sim 55 \%$ of PVR [4]. This was interpreted as being compatible with pathological changes that dominate at the site of the smallest arterioles [4]. A surprise finding was an absolute value for $P_{\mathrm{c}}$ of $\sim 30 \mathrm{mmHg}$, potentially associated with increased capillary filtration. Lung oedema is not known to be a frequent occurrence in patients with $\mathrm{PPH}[5]$.

In the study by KAFI et al. [4], pulmonary artery pressure $(P$ pa $)$ decay curves after arterial occlusion were analysed using a single exponential fitting procedure derived from a simple model of the pulmonary circulation made up of arterial and venous resistances around a capillary capacitance [6]. Theoretically, this approach can be improved using a double exponential fitting based on a more realistic model of the distribution of arterial, capillary and venous resistances and capacitances [7].

It was therefore of interest to compare both methods in a larger number of patients to check whether previously described increased $P_{\mathrm{c}}$ might be a methodological artefact. Not only patients with "pure" PPH, but also with pulmonary arterial hypertension $(\mathrm{PAH})$, as defined by a recent World Health 
Organization-sponsored consensus conference [8], were included. The study sought to determine whether $P$ c, as computed with optimal methodology, might be better correlated with clinical state than traditionally measured $P$ pa and PVR. For the purpose of comparison, $P_{\mathrm{c}}$ was also measured by arterial occlusion in two patients with pulmonary veno-occlusive disease (PVOD) and in four patients with chronic thromboembolic pulmonary hypertension (CTEPH). An increased venous resistance would be expected to increase $P_{\mathrm{c}}$ at a given $P \mathrm{pa}$, and to prolong the $P_{\mathrm{pa}}$ decay curve after occlusion. Conversely, an increase in proximal resistance, as seen in CTEPH, would be expected to shorten the $P$ pa decay curve after occlusion, leading to decreased $P$ c at a given $P$ pa.

\section{Methods}

\section{Patients}

Forty-two patients, 12 males and 31 females aged $52 \pm 14$ yrs (mean \pm SD) gave informed consent to this study. The study was approved by the institutional review boards of the Erasme University Hospital (Brussels, Belgium), and the Gasthuisberg University Hospital (Leuven, Belgium) and the UCSD Medical Center (San Diego, CA, USA) and conformed with the principles outlined in the Declaration of Helsinki. All of the patients had a $P$ pa of $>25 \mathrm{mmHg}$ at rest. Thirty-six patients fitted into the diagnostic criteria of PAH as defined by a recent World Health Organizationsponsored consensus conference that extended the concept of PPH to associated conditions, including collagen vascular diseases, congenital systemic-to-pulmonary shunts, human immunodeficiency virus (HIV) infection, anorexigen intake, and portal hypertension [8]. From those 36 patients, 19 had PPH, 11 had pulmonary hypertension $(\mathrm{PH})$ associated with anorexigens, three had portal $\mathrm{PH}$, and three had $\mathrm{PH}$ associated with scleroderma. Two patients, a 16-yr-old male and a female aged 43 yrs, were suffering from PVOD. This diagnosis rested on radiological signs of interstitial and alveolar oedema, which were markedly aggravated in the male shortly after an attempt at prostacyclin therapy. The diagnosis of PVOD was confirmed in the female by an open lung biopsy. Four patients had an angiographically diagnosed CTEPH. Their pre-operative tracings were included in the present study on the basis of absent or minimal $\mathrm{PH}$, with a mean $P$ pa of $22 \mathrm{mmHg}$ (range $19-26 \mathrm{mmHg}$ ) after surgical thromboendarteriectomy, indicating a predominantly proximal increase in PVR. All of the patients were severely dyspnoeic and in New York Heart Association functional class III. None of the patients were being treated with prostacyclin analogues at the time of right heart catheterisation.

\section{Procedures and measurements}

Right heart catheterisation was performed without premedication, with the patient lying supine and breathing room air. A balloon-tipped, flow-directed, pulmonary catheter (131HF7; Baxter Healthcare Corp., Irvine, CA, USA) was inserted into an internal jugular vein under local anaesthetic and floated, under continuous pressure wave monitoring, into a pulmonary artery to measure $P$ pa, pulmonary artery occluded pressure $(P$ pao $), P_{\mathrm{c}}$ (computed from the $P$ pa decay curve), right atrial pressure $(P$ ra $)$ and pulmonary blood flow (Q). Systemic arterial pressure was determined intermittently by an automated blood pressure cuff. Heart rate was determined from a continuously monitored electrocardiographical lead. Transcutaneous arterial oxyhaemoglobin saturation was continuously monitored by pulse oxymetry.

Pulmonary vascular pressures were measured using disposable transducers (TruWave; Baxter Healthcare Corp.) connected to a bedside haemodynamic and electrocardiographical monitoring system (Sirecust 404; Siemens, Erlangen, Germany). The pressure transducers were zero referenced at mid-chest, and vascular pressures were obtained at end-expiration. $Q$ was measured using the thermodilution technique as a mean of at least three successive measurements (COM-2; Baxter Healthcare Corp.). Inhaled nitric oxide (NO) was supplied from a pure NO source tank (Oxhydrique, Machelen, Belgium) and delivered through a tight facemask. The inspired fraction of NO was monitored by chemiluminescence after calibration against standard NO concentration (42 chemiluminescence NO-NO2Nox analyser; Thermo Environmental Instruments Inc., Franklin, MA, USA). The pulmonary vascular pressure signals were sampled at $200 \mathrm{~Hz}$ using an analogue-to-digital converter (DAS 8-PGA; KeithleyMetrabyte, Taunton, MA, USA), and stored and analysed on a personal computer.

\section{Pulmonary capillary pressure measurements}

$P$ c was computed in triplicate from $P$ pa decay curves after inflation of the balloon of the pulmonary artery catheter. For this measurement, the patients were asked to stop breathing at the end of a normal tidal volume for $10 \mathrm{~s}$. All $P$ pa signals were filtered using a two-pole digital low-pass filter with a cut-off at $18 \mathrm{~Hz}$. The $P$ pa decay curves were analysed by two different methods. First, by fitting the data between $0.2-2$ s post-occlusion with a mono-exponential equation and by deriving the pressure $152 \mathrm{~ms}$ after the moment of occlusion [6,9]. Second, by fitting the data between the moment of occlusion and the stabilisation of the pressure tracing at the level of the $P$ pao with a bi-exponential equation, and by the calculation of $P$ c with the exact solution for a three-compartment model of the pulmonary circulation formalised by BACONNIER et al. [7], with a normalisation procedure to mean $P$ pa $[10]$. PVRa was calculated as $(P \mathrm{pa}-P \mathrm{c}) / \mathrm{Q}$ and expressed as the percentage of PVR, calculated as $(P$ pa $-P$ pao $) / Q$.

\section{Clinical evaluation}

The clinical state of all of the $\mathrm{PAH}$ patients was assessed by a score made up of the following 16 
symptoms and signs: fatigue, dyspnoea, orthopnoea, jugular vein distension, peripheral oedema, syncope, dizziness, palpitation, chest pain, loud second heart sound, third heart sound, fourth heart sound, right ventricular heave, systolic murmur, diastolic murmur and hepatomegaly [11].

\section{Study protocol}

As soon as steady-state conditions (stable heart rate and $P$ pa for $20 \mathrm{~min}$ ) were ensured, a baseline set of haemodynamic measurements was obtained. The measurements were repeated after a 20-min equilibration period under inhalation of 20 parts per million of NO in 22 of the PAH patients. This dose of NO is two times the dose previously shown to offer a maximum pulmonary vasodilating effect in patients with PPH [12].

\section{Statistical analysis}

Results are presented as mean \pm SE. Comparisons of haemodynamic variables at baseline and during inducible NO administrations in the same patients were made by paired t-test. Linear correlations were calculated between haemodynamic variables and the signs and symptoms score.

\section{Results}

As shown in table 1, the pulmonary haemodynamic profile of the PAH patients was similar to that previously reported in large series of patients with PPH, that is, very high $P$ pa, moderately increased $P$ ra, normal $P$ pao and decreased $Q[5,11]$. $P_{c}$ calculated from mono-exponential fitting was increased to $34 \pm 1 \mathrm{mmHg}$, with a PVRa of $48 \pm 2 \%$. $P_{\mathrm{c}}$ calculated from bi-exponential fitting was lower $(\mathrm{p}<0.01)$, but still increased to an average of $26 \pm 1 \mathrm{mmHg}$, with a PVRa of $63 \pm 1 \%$. All further reported $P$ c values were calculated from double exponential fitting procedure. Inhaled NO slightly decreased PVR, with small but significant decreases in $P$ pa and $P$ ra. Inhaled NO did not change the partition of PVR.

The $P$ pa decay after occlusion was prolonged in the patients with PVOD and was shorter in the patients with CTEPH (fig. 1). In comparison with the patients with PAH, those with PVOD had increased $P_{\mathrm{c}}$ and decreased PVRa. In patients with CTEPH, $P_{c}$ was decreased and PVRa increased, but the measurements did not allow a clear discrimination between PAH, PVOD and CTEPH (fig. 2).

In the PAH patients, there was no correlation between $P$ pa, $P$ ra or $P_{\mathrm{c}}$ and the clinical score, while PVR was directly correlated with the clinical score $(\mathrm{r}=0.384, \mathrm{p}<0.05)$. $P$ c was correlated with $P$ pao $(\mathrm{r}=0.79$, $\mathrm{p}<0.001)$ and $P$ pa $(\mathrm{r}=0.76, \mathrm{p}<0.001)$.

\section{Discussion}

The present results show that compared to PAH, PVRa is increased in CTEPH and decreased in PVOD, but that isolated measurement of PVRa does not allow a differential diagnosis between these three types of severe $\mathrm{PH}$.

Measurement of pulmonary capillary pressure versus pulmonary artery occlusion pressure

Inflation of the balloon at the tip of a pulmonary artery catheter to measure $P$ pao creates a downstream stop-flow phenomenon extending to same diameter veins. Therefore, $P$ pao generally gives a satisfactory estimate of left atrial or end-diastolic left ventricular pressure. Wedging a pulmonary artery catheter without balloon inflation yields a pulmonary artery wedge pressure, sometimes called a pulmonary capillary wedge pressure or (wrongly) a pulmonary capillary pressure, which measures the pressure of same diameter veins. Increased venous resistance may increase pulmonary artery wedge pressure relative to $P$ pao. The measurement of an effective pulmonary capillary pressure, $P$ c, requires the analysis of a $P$ pa decay curve after balloon occlusion $[1,2]$.

Table 1.-Haemodynamics at baseline and during inhalation of 20 parts per million nitric oxide (NO) in patients with pulmonary arterial hypertension and at baseline in four patients with chronic thromboembolic pulmonary hypertension (CTEPH) and two patients with pulmonary veno-occlusive disease (PVOD)

\begin{tabular}{|c|c|c|c|c|}
\hline & Baseline & NO & СТЕРН & PVOD \\
\hline Subjects n & 36 & 22 & 4 & 2 \\
\hline HR beats $\cdot \min ^{-1}$ & $78 \pm 2$ & $77 \pm 3^{*}$ & $65 \pm 6$ & 71,89 \\
\hline $\mathrm{Q} \mathrm{L} \cdot \mathrm{min}^{-1} \cdot \mathrm{m}^{-2}$ & $2.0 \pm 0.1$ & $2.2 \pm 0.1$ & $2.1 \pm 0.2$ & $1.5,1.5$ \\
\hline$P$ pa $\mathrm{mmHg}$ & $56 \pm 2$ & $49 \pm 3 * *$ & $47 \pm 5$ & 50,56 \\
\hline Pra $\mathrm{mmHg}$ & $8 \pm 1$ & $6 \pm 1 * *$ & $7 \pm 2$ & 7,8 \\
\hline$P$ pao $\mathrm{mmHg}$ & $11 \pm 1$ & $12 \pm 1$ & $12 \pm 2$ & 12,12 \\
\hline PVR dyn $\cdot \mathrm{s}^{-1} \cdot \mathrm{cm}^{-5} \cdot \mathrm{m}^{-2}$ & $1908 \pm 126$ & $1480 \pm 147 * * *$ & $1352 \pm 218$ & 2082,2347 \\
\hline$P_{\mathrm{c}} \mathrm{mmHg}$ & $26 \pm 1$ & $25 \pm 2$ & $21 \pm 2$ & 34,37 \\
\hline PVRa $\%$ & $63 \pm 1$ & $65 \pm 2$ & $77 \pm 3$ & 42,43 \\
\hline
\end{tabular}

Data are presented as mean \pm SEM unless otherwise stated. HR: heart rate; Q: pulmonary blood flow; $P$ pa: mean pulmonary arterial pressure; $P$ ra: right atrial pressure; $P$ pao: pulmonary artery occluded pressure; PVR: pulmonary vascular resistance; $P$ c: pulmonary capillary pressure; PVRa: arterial component of the PVR. *: $\mathrm{p}<0.05$ compared with baseline; **: $\mathrm{p}<0.01$ compared with baseline; $* * *: \mathrm{p}<0.001$ compared with baseline. 

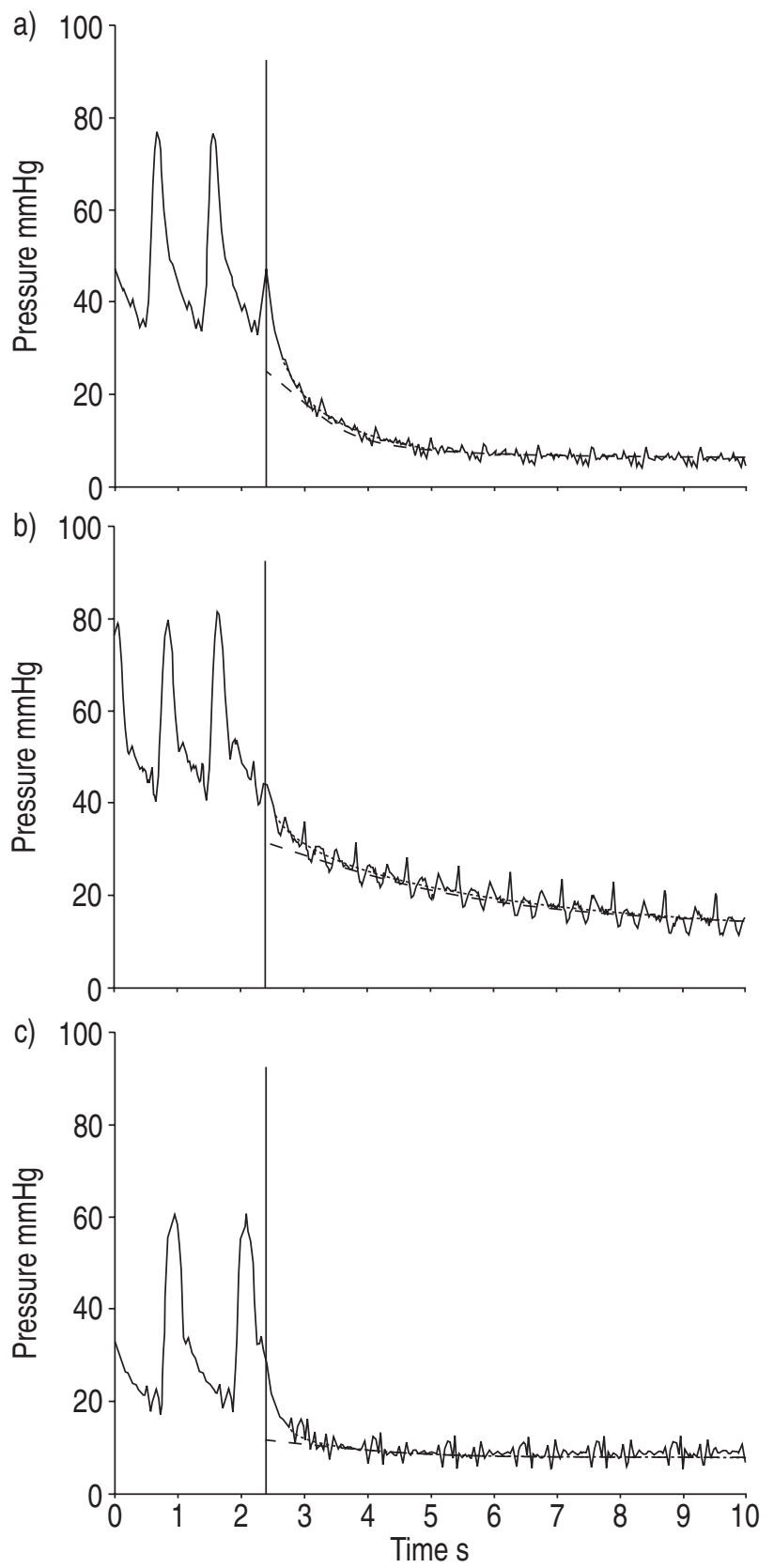

Fig. 1.-Typical pulmonary artery occlusion recordings used to estimate pulmonary capillary pressure $\left(P_{\mathrm{c}}\right)$ in a) a patient with a pulmonary arterial hypertension, b) a patient with pulmonary veno-occlusive disease and c) a patient with chronic thromboembolic pulmonary hypertension. The bi-exponential fittings are shown in dotted lines, and the evolution of $P_{\mathrm{c}}$ after occlusion (vertical solid lines) calculated with the three-compartment model of the pulmonary circulation in dashed lines.

\section{Computing of pulmonary capillary pressure}

Based on pulmonary circulation, modelled as an electrical circuit made of a capillary capacitance between the arterial and venous resistances (R-C-R), the $P$ pa decay curve after balloon occlusion can be fitted with a mono-exponential function, and $P_{\mathrm{c}}$ can be calculated by extrapolation to the moment of occlusion or shortly thereafter [6]. There is, however, a concern that $P_{\mathrm{c}}$ estimated from a mono-exponential
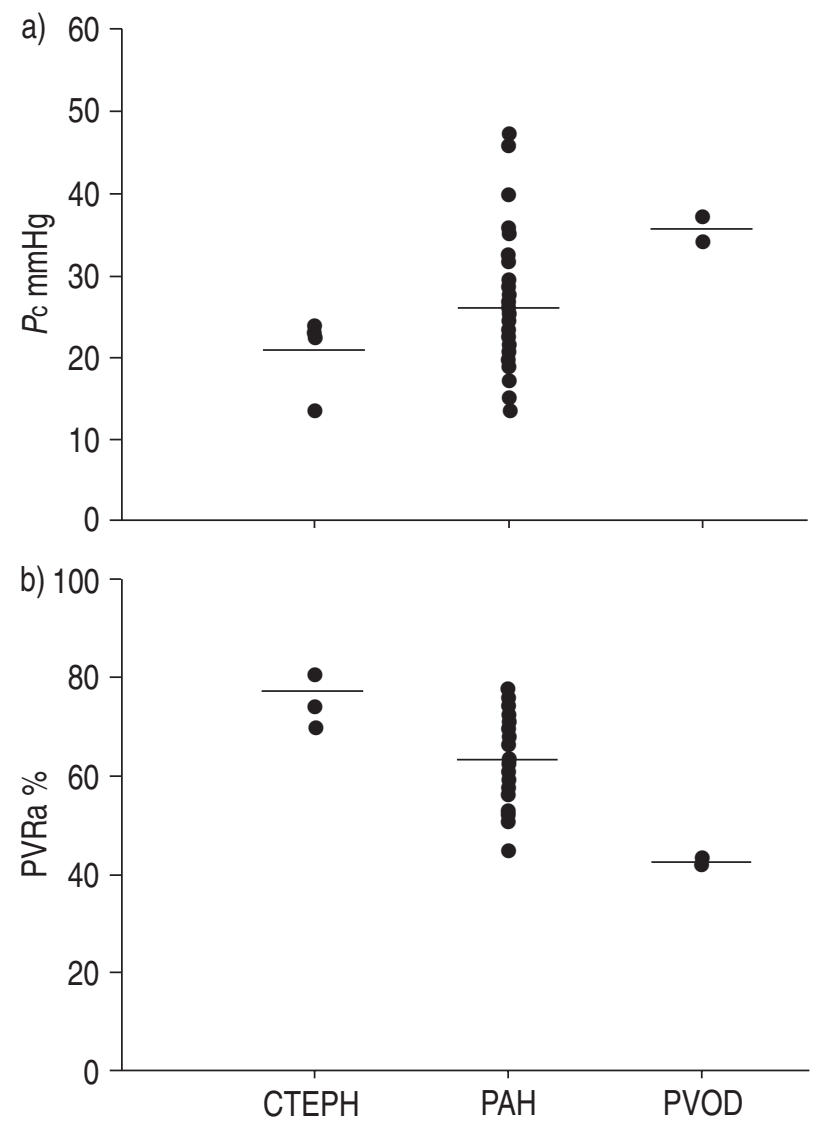

Fig. 2.-a) Pulmonary capillary pressure $\left(P_{\mathrm{c}}\right)$ and b) arterial segment of pulmonary vascular resistance (PVRa) values in patients with pulmonary arterial hypertension (PAH), chronic thromboembolic pulmonary hypertension (CTEPH) and pulmonary veno-occlusive disease (PVOD). The solid line represents the mean value in PAH and CTEPH patients.

fitting might overestimate capillary pressure, as measured by double occlusion or by the reference isogravimetric method [13, 14]. Direct micropuncture measurements compared with single and double occlusion determinations suggest that $P$ c estimated from a mono-exponential fitting may be affected by small arterioles resistance in addition to capillary-venous resistance [15].

A better fit of $P$ pa decay curves after balloon occlusion is obtained with a bi-exponential function [16]. Since both the arterial and venous segments of the pulmonary circulation can be characterised by resistive and compliant properties, BACONNIER et al. [7] formalised a three-compartment C-R-C-R-C model, and proposed an exact solution to compute $P \mathrm{c}$ from the bi-exponential fitting of the $P$ pa decay curve. As there is a phasic variation of $P \mathrm{cc}$ within the cardiac cycle [17], $P_{\mathrm{c}}$ may be better normalised to mean $P$ pa [10]. In the present study, this method of calculation led to lower $P_{\mathrm{c}}$ values than previously found using a mono-exponential fitting in $\mathrm{PPH}$ patients [3, 18]. However, it is uncertain whether any contribution of the smallest size pulmonary arterioles to calculated $P_{\mathrm{c}}$ is excluded.

The present results confirm that $P_{\mathrm{c}}$ computed from mono-exponential fitting is very high in PAH [4]. 
However, $P$ c derived from bi-exponential fitting was still higher than normal. Based on reported measurements of $P$ pa and $P$ pao in normal subjects, and a normal PVRa of $\sim 60 \%$ [3], $P$ c should not normally exceed $16 \mathrm{mmHg}$. $P_{\mathrm{c}}$ values that increased above $20 \mathrm{mmHg}$ could be expected to be associated with an increased extravascular lung water [3]. However, as lung oedema is not known as a usual feature of PAH [5], it is most likely that high $P \mathrm{c}$ measured in PAH would be caused by the effect of increased smallest arteriolar resistance. An alternative, though less likely explanation, would be that the lungs of PAH patients, like patients with left heart failure, would be adapted to chronically increased capillary filtration pressure by capillary remodelling and increased lymphatic flow.

\section{Mechanisms of increased pulmonary capillary pressure in pulmonary arterial hypertension}

Histological studies in PPH show various combinations of medial hypertrophy, concentric or excentric intimal fibrosis, and complex arteritis, plexiform or dilatation lesions in $\sim 30 \%$ of cases, in situ thrombosis [19]. These histological features seem nonspecific for PPH and are described in a variety of secondary forms of PAH, such as $\mathrm{PH}$ associated with anorexigen intake [19], CREST syndrome [20], HIV infection [21] and chronic liver disease [22]. The lesions appear to predominate in small, $<500-1,000 \mu \mathrm{m}$ diameter, arterioles, but it is not entirely clear whether they might extend to more proximal portions of the pulmonary arterial tree, nor whether there might be coexistent lesions of small pulmonary veins.

Spontaneously hypertensive Wistar Kyoto rats that are used as a model of PH show increased muscularity in small pulmonary veins as well as in arteries [23]. In a series of 19 patients with PPH, intimal and/or advential increased thickness of the pulmonary venous walls was found in half of them [24]. Venous involvement is also present in PH associated with CREST syndrome [20]. It has been estimated that $5-25 \%$ of patients with clinically diagnosed PPH present with predominant venoocclusive lesions at careful pathological examination of biopsic or autopsic specimens [25]. PVOD has also been described in patients infected with HIV [21]. Therefore, the present finding of increased absolute values of $P_{\mathrm{c}}$ with a "normal" longitudinal distribution of the PVR, may reflect that venous involvement is more important than previously assumed in patients with PAH. Detailed morphological studies will be needed to assess whether the variability of recorded $P_{\mathrm{c}}$ in the present study might reflect variability in this venous involvement in PAH.

\section{Effects of inhaled nitric oxide in pulmonary arterial hypertension}

These data show that inhaled NO decreases PVR without changing its partition. Inhaled NO has been reported in isolated perfused lungs of various species after different vasoconstrictor stimuli either to unalter the longitudinal distribution of resistances [26] or to act predominantly at precapillary level [27]. Inhaled
NO did not affect the longitudinal distribution of resistances in experimental micro-embolic PH [28] but decreased the capillary-venous component of PVR in acute respiratory distress syndrome [29]. Inhaled NO diffuses easily through the alveolo-capillary membrane before its inactivation by haemoglobin, and probably dilates the smallest arterioles and venules adjacent to the alveolar space, provided there is a component of active constriction. These results confirm that in most patients with $\mathrm{PAH}$, this component of vasoconstriction is minimal, and that inhaled NO dilates both arterioles and venules.

\section{Clinical and haemodynamic correlations}

In the present study, the signs and symptoms score was correlated with PVR and not $P$ pa or $P$ c. This is in keeping with previous studies showing that PVR or Q are better correlated with exercise or functional capacity in PAH patients than $P$ pa [30]. There were significant correlations between $P$ c and $P$ pa or $P$ pao. This is explained by upstream transmission of left heart filling pressures, and by the fact that increased capillary-venous resistance necessarily increases both $P$ pa and $P$.

\section{Clinical implications}

There is only one previously reported patient with PVOD and $P$ c measured using the occlusion method [31]. The present results do not agree with that study's suggestion that an increased $P_{\mathrm{c}}$ is diagnostic of PVOD.

Only two patients with PVOD could be included in the present study, precluding meaningful statistical comparisons with patients with PAH. This is explained by the fact that the disease is very rare, with an estimated incidence of $0.1-0.2$ cases per million persons per year [25]. In addition, these patients are often critically ill and unstable at the time of diagnosis, making the collection of sufficient quality arterial occlusion data problematic.

Most patients with CTEPH present with some degree of persistent $\mathrm{PH}$ after successful thromboendarteriectomy [32]. In this study, a small series of four such patients, selected for minimal residual $\mathrm{PH}$, had a $P_{\mathrm{c}}$ less elevated on average than in patients with PAH, but still higher than normal, suggestive of peripheral small vessel involvement. Whether the occlusion technique has the potential of helping the identification of patients with CTEPH at risk of postoperative $\mathrm{PH}$ is currently under investigation, with promising preliminary results [33].

\section{Conclusions}

In patients with pulmonary arterial hypertension, pulmonary capillary pressure measured with the occlusion technique is higher than normal and may be due to a previously assumed unimportant venous involvement. The single arterial occlusion technique helps to locate the site of predominantly increased pulmonary vascular resistance in severe pulmonary hypertension, but does not discriminate between pulmonary 
arterial hypertension, pulmonary veno-occlusive disease and chronic thromboembolic pulmonary hypertension.

Acknowledgements. The authors thank the staff of the Intensive Care Unit and the staff of the Coronary Care Unit of the Erasmus University Hospital (Brussels, Belgium) for their help with patient care. M-T. Gautier and P. Jespers helped in the preparation of this report.

\section{References}

1. Cope DK, Allison RC, Parmentier JL, Miller JN, Taylor AE. Measurement of effective pulmonary capillary pressure using the pressure profile after pulmonary artery occlusion. Crit Care Med 1986; 14: 16-22.

2. Grimbert FA. Effective pulmonary capillary pressure. Eur Respir J 1988; 1: 297-301.

3. Maggiorini M, Mélot C, Pierre S, et al. High altitude pulmonary edema is initially caused by an increased capillary pressure. Circulation 2001; 103: 2078-2083.

4. Kafi SA, Mélot C, Vachiery JL, Brimioulle S, Naeije R. Partitioning of pulmonary vascular resistance in primary pulmonary hypertension. J Am Coll Cardiol 1998; 31: 1372-1376.

5. Rich S, Dantzker DR, Ayres SM, et al. Primary pulmonary hypertension. A national prospective study. Ann Intern Med 1987; 107: 216-223.

6. Gilbert E, Hakim TS. Derivation of pulmonary capillary pressure from arterial occlusion in intact conditions. Crit Care Med 1994; 22: 986-993.

7. Baconnier PF, Eberhard A, Grimbert FA. Theoretical analysis of occlusion techniques for measuring pulmonary capillary pressure. J Appl Physiol 1992; 73: 1351-1359.

8. Fishman AP. Clinical classification of pulmonary hypertension. Clin Chest Med 2001; 22: 385-391.

9. Hakim TS, Maarek JM, Chang HK. Estimation of pulmonary capillary pressure in intact dog lungs using the arterial occlusion technique. Am Rev Respir Dis 1989; 140: 217-224.

10. Pagnamenta A, Bouckaert Y, Wauthy P, Brimioulle S, Naeije R. Continuous versus pulsatile pulmonary hemodynamics in canine oleic acid lung injury. $A m$ J Respir Crit Care Med 2000; 162: 936-940.

11. Simonneau G, Barst RJ, Galie N, et al. Continuous subcutaneous infusion of treprostinil, a prostacyclin analogue, in patients with pulmonary arterial hypertension. Am J Respir Crit Care Med 2002; 162: 800-804.

12. Sitbon O, Brenot F, Denjean A, et al. Inhaled nitric oxide as a screening vasodilator agent in primary pulmonary hypertension. A dose-response study and comparison with prostacyclin. Am J Respir Crit Care Med 1995; 151: 384-389.

13. Townsley MI, Korthuis RJ, Rippe B, Parker JC, Taylor AE. Validation of double vascular occlusion method for Pc,i in lung and skeletal muscle. $J$ Appl Physiol 1986; 61: 127-132.

14. Maarek JM, Hakim TS, Chang HK. Analysis of pulmonary arterial pressure profile after occlusion of pulsatile blood flow. J Appl Physiol 1990; 68: 761-769.

15. Hakim TS, Kelly S. Occlusion pressures vs. micropipette pressures in the pulmonary circulation. $J$ Appl Physiol 1989; 67: 1277-1285.

16. Dawson CA, Bronikowski TA, Linehan JH, Haworth ST, Rickaby DA. On the estimation of pulmonary capillary pressure from arterial occlusion. Am Rev Respir Dis 1989; 140: 1228-1236.

17. Yamada Y, Suzukawa M, Chinzei M, et al. Phasic capillary pressure determined by arterial occlusion in intact dog lung lobes. J Appl Physiol 1989; 67: 2205-2211.

18. Gaar KA Jr, Taylor AE, Owens LJ, Guyton AC. Pulmonary capillary pressure and filtration coefficient in the isolated perfused lung. Am J Physiol 1967; 213: 910-914.

19. Pietra GG. The pathology of pulmonary hypertension. In: Rubin LJ, Rich S, eds. Primary Pulmonary Hypertension. New York, Marcel Dekker, 1997; pp. 19-61.

20. Yousem SA. The pulmonary pathologic manifestations of the CREST syndrome. Hum Pathol 1990; 21: 467-474.

21. Mesa RA, Edell ES, Dunn WF, Edwards WD. Human immunodeficiency virus infection and pulmonary hypertension: two new cases and a review of 86 reported cases. Mayo Clin Proc 1998; 73: 37-45.

22. Schraufnagel DE, Kay JM. Structural and pathologic changes in the lung vasculature in chronic liver disease. Clin Chest Med 1996; 17: 1-15.

23. Aharinejad S, Schraufnagel DE, Bock $\mathrm{P}$, et al. Spontaneously hypertensive rats develop pulmonary hypertension and hypertrophy of pulmonary venous sphincters. Am J Pathol 1996; 148: 281-290.

24. Chazova I, Loyd JE, Zhdanov VS, Newman JH, Belenkov Y, Meyrick B. Pulmonary artery adventitial changes and venous involvement in primary pulmonary hypertension. Am J Pathol 1995; 146: 389-397.

25. Mandel J, Mark EJ, Hales CA. Pulmonary venoocclusive disease. Am J Respir Crit Care Med 2000; 162: 1964-1973.

26. Lindeborg DM, Kavanagh BP, van Meurs K, Pearl RG. Inhaled nitric oxide does not alter the longitudinal distribution of pulmonary vascular resistance. J Appl Physiol 1995; 78: 341-348.

27. Rimar S, Gillis CN. Site of pulmonary vasodilation by inhaled nitric oxide in the perfused lung. $J$ Appl Physiol 1995; 78: 1745-1749.

28. Mélot C, Vermeulen F, Maggiorini M, Gilbert E, Naeije R. Site of pulmonary vasodilation by inhaled nitric oxide in microembolic lung injury. Am J Respir Crit Care Med 1997; 156: 75-85.

29. Rossetti M, Guenard H, Gabinski C. Effects of nitric oxide inhalation on pulmonary serial vascular resistances in ARDS. Am J Respir Crit Care Med 1996; 154: $1375-1381$.

30. Miyamoto S, Nagaya $\mathrm{N}$, Satoh $\mathrm{T}$, et al. Clinical correlates and prognostic significance of six-minute walk test in patients with primary pulmonary hypertension. Comparison with cardiopulmonary exercise testing. Am J Respir Crit Care Med 2000; 161: 487-492.

31. Davis LL, deBoisblanc BP, Glynn CE, Ramirez C, Summer WR. Effect of prostacyclin on microvascular pressures in a patient with pulmonary veno-occlusive disease. Chest 1995; 108: 1754-1756.

32. Moser KM, Auger WR, Fedullo PF, Jamieson SW. Chronic thromboembolic pulmonary hypertension: clinical picture and surgical treatment. Eur Respir $J$ 1992; 5: 334-342.

33. Kim HS, Fesler P, Channick RN, Naeije R, Rubin LJ. Pre-operative pulmonary artery occlusion measurements correlate with hemodynamic outcome following pulmonary thromboendarteriectomy. Am $J$ Respir Crit Care Med Suppl 2002; 165: A24. 